\title{
Fast computation of the geoelectric field using the method of elementary current systems and planar Earth models
}

\author{
A. Viljanen ${ }^{1}$, A. Pulkkinen ${ }^{1}$, O. Amm ${ }^{1}$, R. Pirjola ${ }^{1}$, T. Korja ${ }^{2, *}$, and BEAR Working Group ${ }^{3}$ \\ ${ }^{1}$ Finnish Meteorological Institute, Geophysical Research Division, P.O.B. 503, FIN-00101 Helsinki, Finland \\ ${ }^{2}$ Geological Survey of Finland, P.O.B. 96, FIN-02151 Espoo, Finland \\ ${ }^{3}$ Contact person: T. Korja \\ *now at: University of Oulu, Department of Geosciences, P.O.B. 3000, FIN-90014 Oulu, Finland
}

Received: 25 October 2002 - Revised: 3 June 2003 - Accepted: 18 June 2003 - Published: 1 January 2004

\begin{abstract}
The method of spherical elementary current systems provides an accurate modelling of the horizontal component of the geomagnetic variation field. The interpolated magnetic field is used as input to calculate the horizontal geoelectric field. We use planar layered (1-D) models of the Earth's conductivity, and assume that the electric field is related to the local magnetic field by the plane wave surface impedance. There are locations in which the conductivity structure can be approximated by a 1-D model, as demonstrated with the measurements of the Baltic Electromagnetic Array Research project. To calculate geomagnetically induced currents (GIC), we need the spatially integrated electric field typically in a length scale of $100 \mathrm{~km}$. We show that then the spatial variation of the electric field can be neglected if we use the measured or interpolated magnetic field at the site of interest. In other words, even the simple plane wave model is fairly accurate for GIC purposes. Investigating GIC in the Finnish high-voltage power system and in the natural gas pipeline, we find a good agreement between modelled and measured values, with relative errors less than $30 \%$ for large GIC values.
\end{abstract}

Key words. Geomagnetism and paleomagnetism (geomagnetic induction; rapid time variations) - Ionosphere (electric field and currents)

\section{Introduction}

Temporal variations of the geomagnetic field are accompanied by an electric field whose horizontal component can, in extreme cases, exceed $10 \mathrm{~V} / \mathrm{km}$ at the Earth's surface. The geoelectric field produces currents in the conducting Earth as well as in manmade conductors such as power systems and pipelines. In the case of technological systems, the term geomagnetically induced current (GIC) is used. GICs are of practical importance due to possibly harmful effects (Bolduc, 2002; Boteler et al., 1998; Gummow, 2002; Kap-

Correspondence to: A. Viljanen (ari.viljanen@ fmi.fi) penman, 1996; Lahtinen and Elovaara, 2002; Lesher et al., 1994; Molinski, 2002; Osella et al., 1998; Pirjola et al., 2002). Only few really severe GIC disturbances have occurred, but developing methods for calculating the geoelectric field is relevant as basic research, too: a successful modelling of GIC requires a detailed knowledge of ionospheric currents and of the Earth's conductivity, and provides a comprehensive test for geospace models.

The calculation of GIC in a conductor system consists of two independent steps: determination of the geoelectric field, and computation of GIC due to the given electric field. The latter task is different for discretely (e.g. power grids; see Lehtinen and Pirjola, 1985) and continuously grounded systems (e.g. buried pipelines; see Pulkkinen et al., 2001a; Trichtchenko and Boteler, 2002), but it is simple compared to the former part. The difficulty in the geophysical step arises from the fact that GIC is not only affected by spatially and temporally complicated ionospheric currents, but the geoelectric field also crucially depends on the Earth's conductivity. In this sense the electric field differs from the magnetic field, because the latter can be fairly accurately calculated without taking into account induction effects (cf. Tanskanen et al., 2001).

The calculation of the geoelectric field requires that ionospheric currents are known as functions of time and space, and that a model of the Earth's conductivity is known. In practice, a full 3-D modelling of the whole geospace is not feasible. The knowledge of the ionosphere and the Earth is never perfect either. In GIC studies it is not necessary to know the spatial structure of the electric field in a kilometre scale. When GIC is determined in a power system or a pipeline, the electric field is integrated along the conductors. Consequently, the relevant spatial scale is given, for example, by the distances between nodes of a power system, which are at least tens of kilometres in Finland. Consequently, regional averages of the field are adequate for GIC purposes.

A sophisticated computation technique with layered conductivity structures is the complex image method (CIM) (Lindell et al., 2000; Thomson and Weaver, 1975; Wait and 
Spies, 1969), which, in a generalised form, allows for including realistic 3-D models of ionospheric currents (Pirjola and Viljanen, 1998). It allows for use of closed-form formulas, thus making computations much faster than with the exact Fourier integrals. Viljanen et al. (1999a) and Viljanen et al. (1999b) applied this technique to models of typical ionospheric events in order to investigate the magnetotelluric source effect, and to calculate GIC in the Finnish power grid.

A major advantage in the modelling of the ionosphere is the method of spherical elementary current systems (SECS) (Amm and Viljanen, 1999; Pulkkinen et al., 2003a,b). It allows for rapid determination of equivalent ionospheric currents from regional to global scales. The combination of CIM and SECS was introduced by Pulkkinen et al. (2003a), and we now demonstrate its applicability of determining the geoelectric field for GIC calculations. Although CIM is a convenient tool for theoretical modelling purposes, it is not the most optimal choice for more operational applications. As a replacement, we show that a simple local 1-D assumption is very reasonable when we aim at calculating the spatially averaged geoelectric field from the magnetic field determined with SECS. So we can calculate the electric field at any surface point with a different 1-D conductivity model at each site if desired, and then numerically integrate the field along conductors to obtain the GIC. We also show that even the simplest plane wave method with spatially uniform fields is good enough in GIC calculations, on the important condition that the magnetic field used is measured close to or interpolated at the GIC site under study. A further advantage in the plane wave method is that GIC is directly determined by the horizontal electric field via constant multipliers without the need to integrate the field separately for each time step.

We present a strict physical approach, which starts from geomagnetic recordings and provides the geoelectric field at any point at the Earth's surface. Our method is well suited to post-analysis of interesting events. In addition, it would be useful for predicting the geoelectric field, provided that forecasts of ground magnetic field variations are available. Although this paper focuses on the methodology, it also shows how complicated the geometry of the geoelectric field is and how rapidly it changes. Pulkkinen et al. (2003c) present a more detailed analysis about ionospheric currents during an extreme event.

After briefly reviewing some central equations, we first show that the assumption of a planar geometry is reasonable. Then we demonstrate that the time derivative of the ground magnetic field can be reproduced with a good accuracy, even with quite a sparse magnetometer network. This is a critical point when considering the geoelectric field. Usefulness of the local 1-D assumption during highly disturbed events is shown by comparing modelled and measured data of the electric field. The most important evidence is given by comparing measured and modelled GIC in the Finnish highvoltage power system and in the natural gas pipeline.

\section{Method of calculation}

Equivalent ionospheric currents are determined using spherical elementary current systems, as described by Amm and Viljanen (1999), and validated in detail by Pulkkinen et al. (2003a). The method is based on the fact that geomagnetic variations at the Earth's surface can be explained by a horizontal divergence-free current system at the ionospheric level. Strictly speaking, induction effects in the Earth should be included by setting another current layer below the Earth's surface, but it is omitted in this study. We are interested in highly disturbed events, where the ionospheric contribution to horizontal magnetic variations close to strong currents is typically more than $80 \%$ of the total variation (Tanskanen et al., 2001). Neglecting induction effectively means that we obtain slightly overestimated amplitudes of ionospheric equivalent currents, but the geometric patterns of the current systems remain practically unchanged. It is possible to separate the field into external and internal parts with SECS (Pulkkinen et al., 2003b), but at regions with sparse measurements this would not lead to a significantly better result.

We cannot determine the true 3-D ionospheric current system by using ground magnetometer data only. However, for any given 3-D system, a horizontal equivalent current system exists that produces the same magnetic and electric field at the Earth's surface (cf. Pirjola and Viljanen, 1998). Furthermore, as will be seen, we do not actually need ionospheric equivalent currents, but just the total horizontal variation field at the Earth's surface.

Amplitudes of elementary current systems are determined by fitting the modelled horizontal field to the measured one. Although this is done in a spherical geometry, we can assume a planar geometry in local applications, which makes computations much simpler without affecting the modelled fields too much. Then the surface current density of an elementary system with an amplitude $I$ at height $h$ in cylindrical coordinates is $\left(r=\sqrt{x^{2}+y^{2}}\right)$ is $\mathbf{J}(r)=I /(2 \pi r) \mathbf{e}_{\phi}$, as given by Amm (1997) (misprint in his formula corrected here). The electric field at the Earth's surface due to one element is

$\mathbf{E}=-\frac{i \omega \mu_{0} I}{4 \pi} \frac{\sqrt{r^{2}+h^{2}}-h}{r} \mathbf{e}_{\phi}$,

where the $z$ axis points vertically downwards, and the Earth's surface is the $x y$ plane, and a harmonic time-dependence $\left(e^{i \omega t}\right)$ is assumed (e.g. Pulkkinen et al., 2003a). The magnetic field is

$\mathbf{B}=\frac{\mu_{0} I}{4 \pi r}\left(\left(1-\frac{h}{\sqrt{r^{2}+h^{2}}}\right) \mathbf{e}_{r}+\frac{r}{\sqrt{r^{2}+h^{2}}} \mathbf{e}_{z}\right)$.

Because the field is calculated directly from a given current system, it automatically fulfills the Maxwell equations, especially the curl-free condition of the magnetic field outside of the source region. So the SECS method provides a robust interpolation technique.

In the complex image method the effect of the Earth is approximated by setting a perfect conductor at the complex depth $p(\omega)=Z(\omega) /\left(i \omega \mu_{0}\right)$, where $Z(\omega)$ is the plane wave 
surface impedance of the layered Earth defined by the thicknesses and electromagnetic parameters of the layers. To calculate the field due to induced currents in the Earth, $h$ in Eq. (1) is replaced by $h+2 p(\omega)$, and the signs of $B_{z}$ and $\mathbf{E}$ are changed (corresponding to the opposite sign of the image current). So induction tends to decrease the horizontal electric field and the vertical magnetic field, and to increase the horizontal magnetic field.

The simplest method to calculate the electric field from magnetic data is the local 1-D model, when

$$
E_{x}(\omega)=\frac{Z(\omega)}{\mu_{0}} B_{y}(\omega), E_{y}(\omega)=-\frac{Z(\omega)}{\mu_{0}} B_{x}(\omega) .
$$

At first sight, this may appear as a severe oversimplification, since it basically requires that the fields are laterally constant, which, in turn, presumes that neither the ionospheric currents, nor the Earth's conductivity have lateral variations. However, the formula works quite well if we use local 1-D Earth models and the local magnetic field. In fact, a strict lateral constancy is not required, but a linear spatial variation of the fields is allowed (Dmitriev and Berdichevsky, 1979).

An advantage compared to CIM is that now the magnetic field provided by SECS is exactly the one needed in the local 1-D method, i.e. the total horizontal variation field at the Earth's surface. So induction effects are not taken into account twice, as in the combination of SECS and CIM, where ionospheric equivalent currents are biased by the effect of telluric currents.

\section{Application to real data}

\subsection{Modelled and measured fields during the BEAR project}

Baltic Electromagnetic Array Research (BEAR) is a subproject of SVEKALAPKO (SVEcofennian-KArelia-LAPlandKOla). SVEKALAPKO is aimed to determine the geometry, thickness and age of the lithosphere, the uppermost shell of the solid Earth, and the disposition of major lithospheric structures in the Fennoscandian (Baltic) Shield (Korja et al., 2002). Within the BEAR measurement phase, June-July 1998, the magnetic and electric fields were recorded with a 2 or 10 -second time resolution in a dense network in Fennoscandia (Fig. 1). In this study, we use oneminute averaged BEAR data.

The elementary current systems were placed in the ionosphere at the height of $100 \mathrm{~km}$ in a regular grid, which covered the continental part of IMAGE with extensions of some degrees. A typical number of grid points was around 250 in the range of 55-75 deg $\mathrm{N}$ and 0-35 deg long, with spacings of $1.25 \mathrm{deg}$ latitudinally and $2.5 \mathrm{deg}$ longitudinally.

We first show that the assumption of a planar geometry is acceptable. Figure 2 depicts the calculated magnetic field in spherical and planar geometries. The amplitudes of elementary current systems were determined in the spherical geometry. The planar geometry was then constructed by a stereographic projection. In particular, the good correspondence

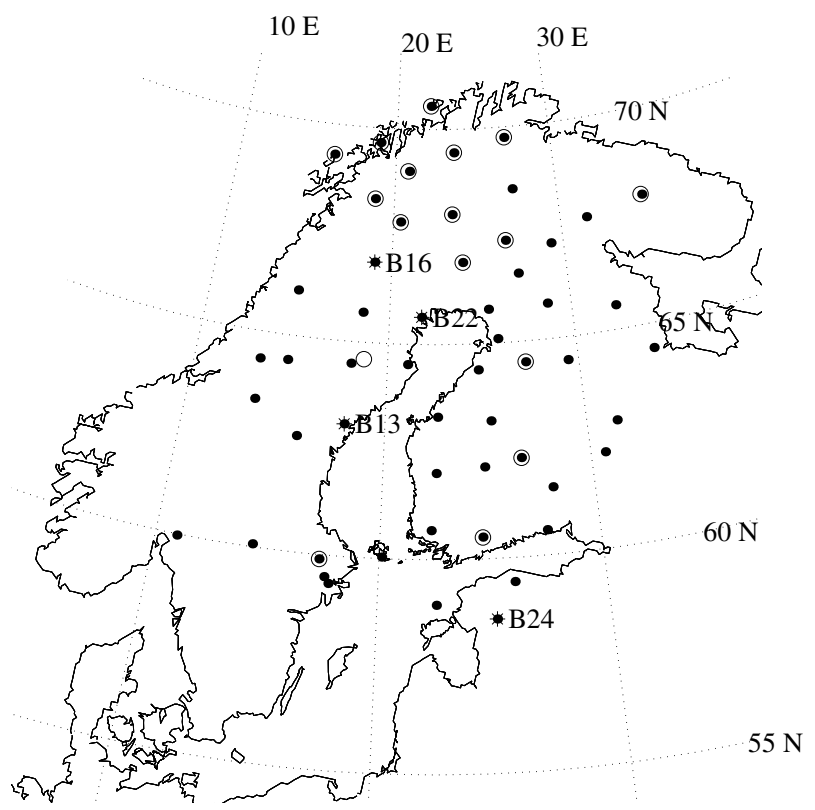

Fig. 1. BEAR sites in summer 1998. IMAGE magnetometer stations operating at that time are marked with circles (Svalbard excluded). The four stations B13, B16, B22, B24 used in Fig. 5 are assigned by their codes.

between the time derivative of the magnetic field is notable, since it is closely related to the geoelectric field. (The time derivative is calculated as a difference between two successive field values.)

Using BEAR data, Pulkkinen et al. (2003a) showed that the SECS method reproduces the horizontal ground magnetic variation field very well, and they also demonstrated that its time derivative $(d \mathbf{H} / d t)$ is accurately reproduced at the measuring sites. We will now have a closer look at $d \mathbf{H} / d t$. To show that the relatively sparse IMAGE network can yield good interpolated values of $d \mathbf{H} / d t$, we consider a disturbed day of 26 June 1998. We determined equivalent currents using the 14 IMAGE magnetometer stations that were operating on the continent during that day. (Nowadays, the situation is better, since there are 22 continental sites.) During an intense substorm, a westward electrojet covered the whole continental part of BEAR, as seen in Fig. 3. There are no striking features in the horizontal field pattern. On the contrary, $d \mathbf{H} / d t$ is much more structured, which is typical at these latitudes (Pulkkinen et al., 2003a,c).

A sequence of $d \mathbf{H} / d t$ plots shows how rapidly the patterns change in a one-minute timescale (Fig. 4). The equivalent currents are not shown, but they are very similar to those in Fig. 3. Recalling that we used only IMAGE magnetometers to determine elementary currents systems, we still obtain a satisfactory fit even for $d \mathbf{H} / d t$. There are clear deviations in magnitudes and direction in places, but the overall structure is mostly well reproduced. As Pulkkinen et al. (2003a) showed, very small-scale ionospheric features (less 

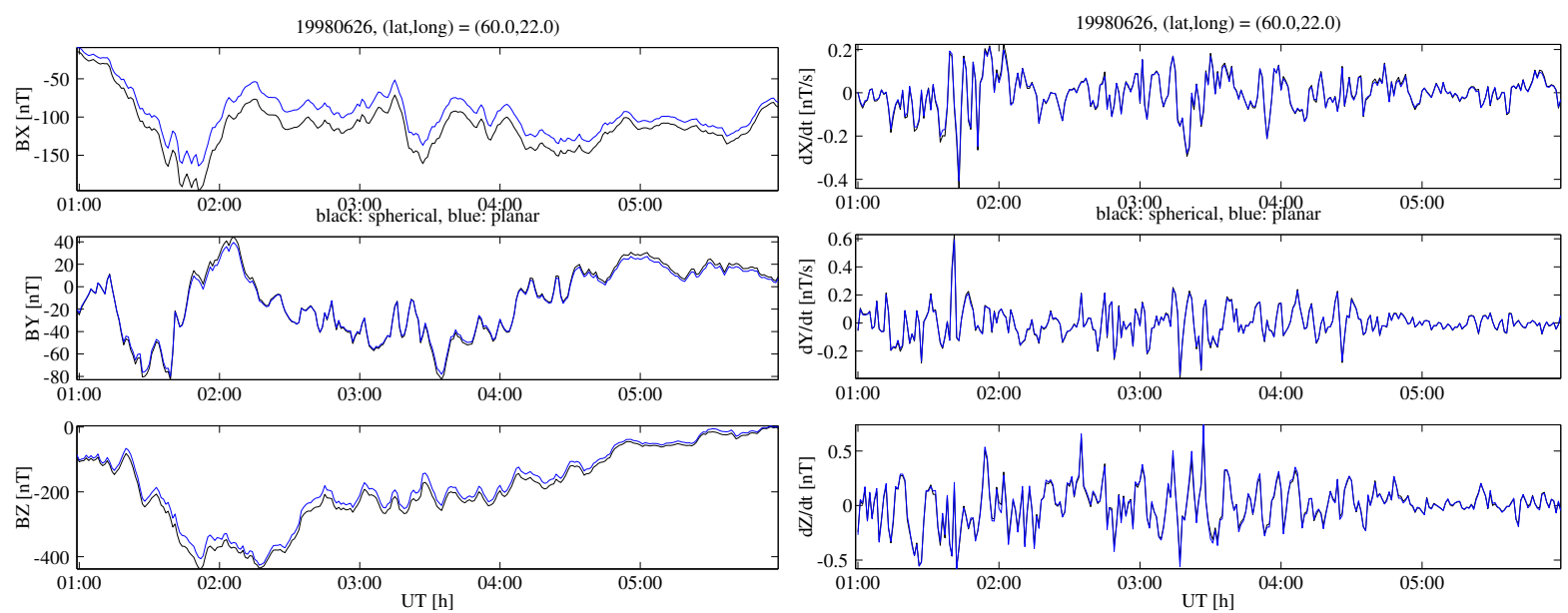

Fig. 2. Comparison of the ground magnetic field and its time derivative calculated in a spherical (black line) and planar geometry (blue line). The ionospheric equivalent current system used as input for both cases was determined in the spherical geometry. The effect of the conducting Earth is ignored.
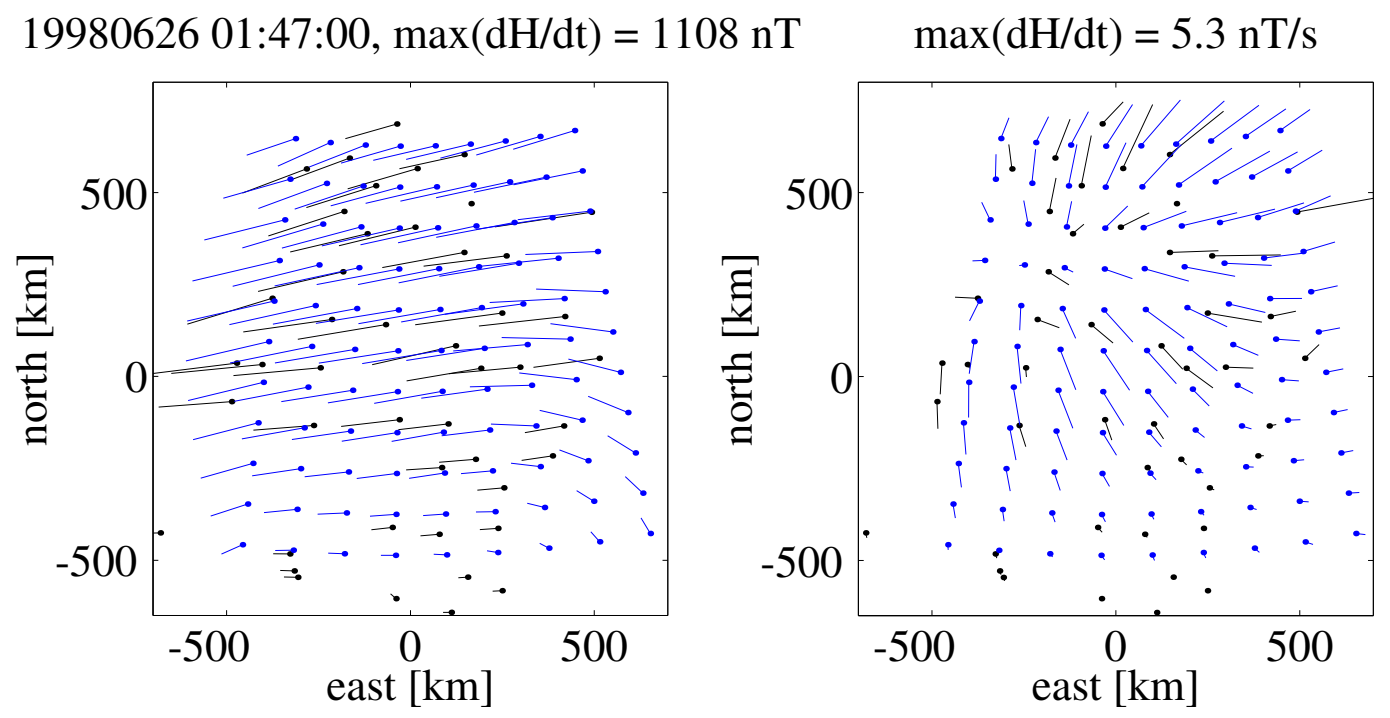

Fig. 3. Measured and modelled horizontal magnetic field in the BEAR region on 26 June 1998, 01:47 UT. Left panel: horizontal magnetic field vectors (rotated 90 degrees clockwise). Right panel: time derivative of the horizontal magnetic field. Measured values are shown with black arrows and modelled with blue arrows. The effect of the conducting Earth is ignored. Only IMAGE magnetometer were used to derive ionospheric equivalent currents.

than about $100 \mathrm{~km}$ scale sizes) cannot be explained due to the sparsity of the ground magnetometer array, and also due to the $100 \mathrm{~km}$ distance from the surface to the ionosphere. It would be possible to improve the fit at measuring sites, but the price would be to allow unrealistically varying patterns between observation points.

The use of the recorded BEAR electric field is more complicated, because it is typically very much distorted due to local geology. However, some stations are obviously at locations where a 1-D Earth model is a good approximation. We calculated the electric field using the local 1-D method with conductivity models compiled from the conductance map of the Fennoscandian Shield (Korja et al., 2002), and averaged on an area of $50 \mathrm{~km} \times 50 \mathrm{~km}$ (Table 1). Examples of four sites at different latitudes during high magnetic activity are shown in Fig. 5. It is noteworthy how accurately the field can be modelled with the local 1-D method, although this is definitely not a plane wave event. The approximative nature of the 1-D assumption is clear, especially at B24. There are several other sites not shown here where the local 1-D model 

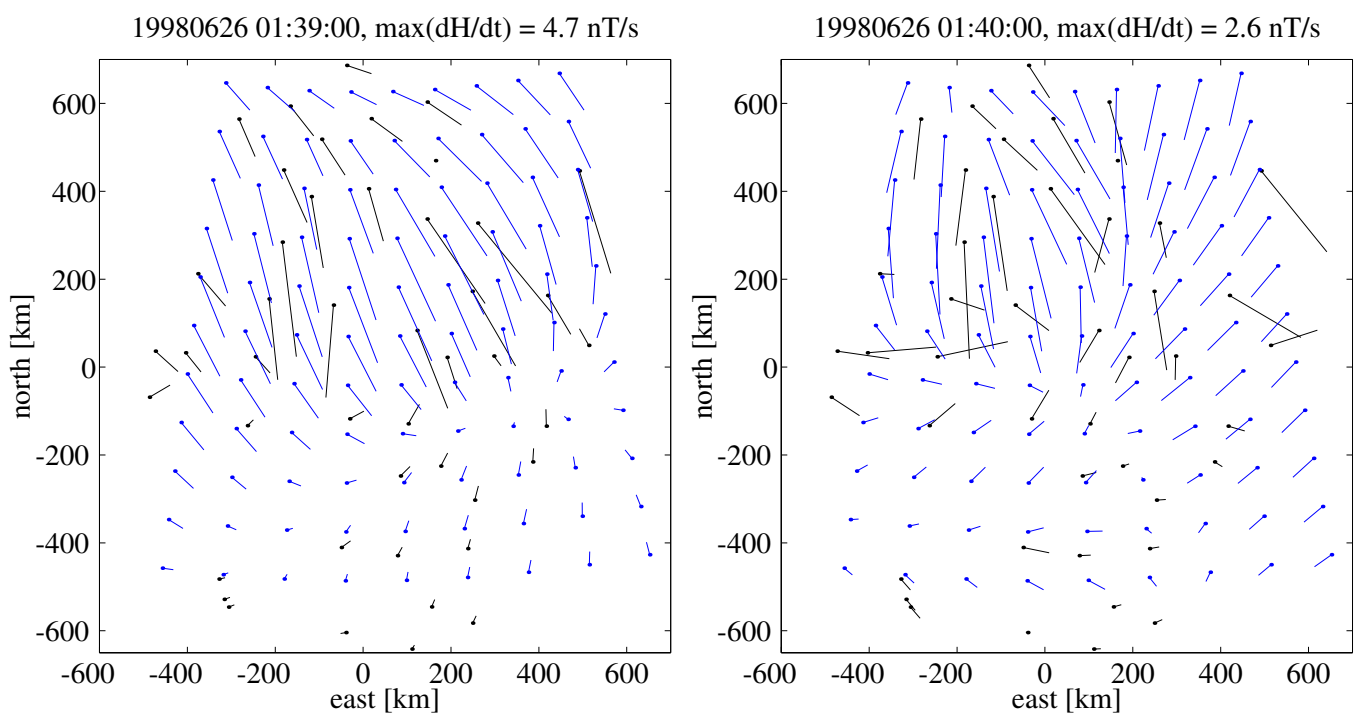

$1998062601: 41: 00, \max (\mathrm{dH} / \mathrm{dt})=2.5 \mathrm{nT} / \mathrm{s}$
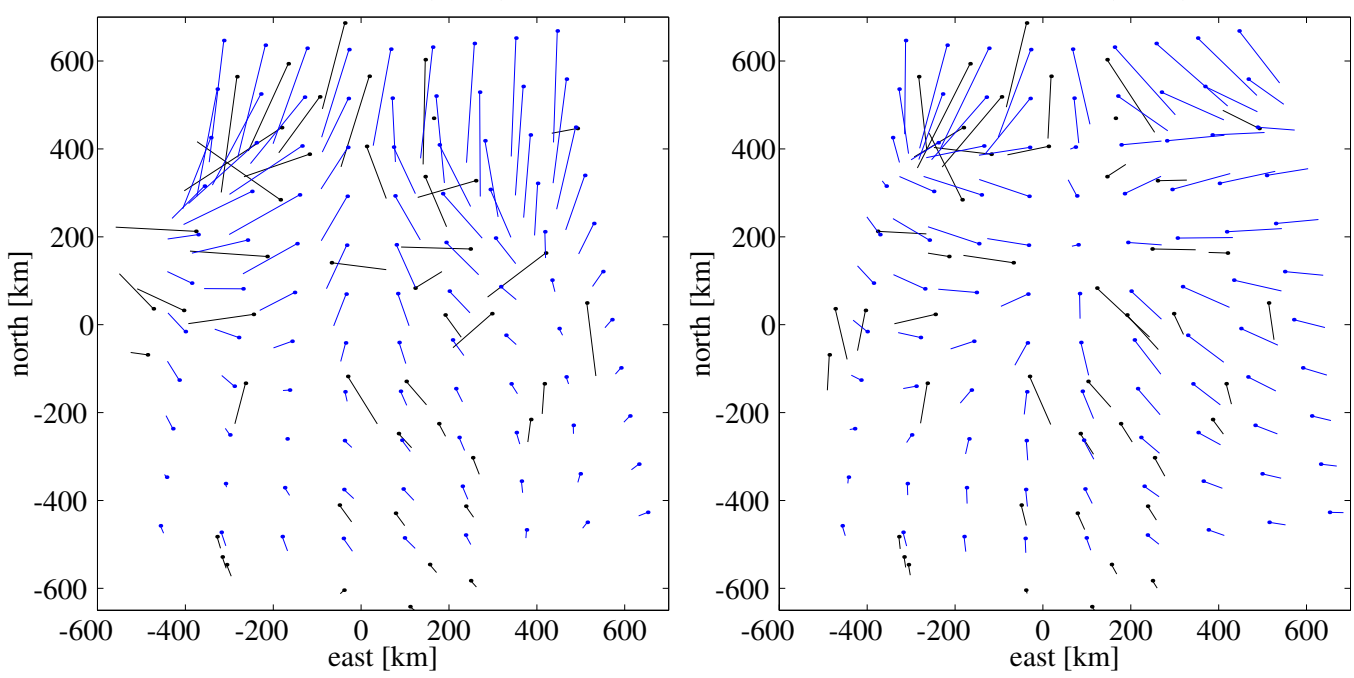

Fig. 4. As Fig. 3, but for time-derivatives of the magnetic field at four consecutive minute intervals in the BEAR region on 26 June 1998.

cannot satisfactorily reproduce the field. However, in GIC studies we need regional averages of the electric field, so a pointwise measured electric field is not very useful. This will become obvious in the next section when comparing measured and modelled GIC.

\subsection{Geomagnetically induced currents}

\subsubsection{Finnish high-voltage power system}

We will first show that CIM can be replaced with the local 1-D method. Using ionospheric equivalent current systems, we calculated the magnetic $\left(\mathbf{B}_{C I M}\right)$ and electric field $\left(\mathbf{E}_{C I M}\right)$ at the Earth's surface with the conductivity model in Table 2 for the whole area, although it is most appropriate for southern Finland (Viljanen et al., 1999a,b). The aim is not to validate Earth models, but to prove that the combination of SECS and the local 1-D method provides reasonable results in GIC modelling, in which we need the electric field smoothed by a spatial integration.

We computed the sum of GIC due to $\mathbf{E}_{C I M}$ at all nodes of the Finnish high-voltage power system. Second, we used $\mathbf{B}_{C I M}$ as input in the local 1-D method with the same Earth model as with CIM and calculated again the sum of GIC. As Fig. 6 indicates, the local 1-D method yields practically the same result as CIM, even during very disturbed events. Consequently, CIM can be replaced with the local 1-D method, which makes the computation faster. Furthermore, as discussed earlier, CIM has the problem of using biased ionospheric equivalent currents affected by telluric currents. In the local 1-D method, the input must be the total horizontal variation field, which is very accurately produced by SECS. The apparent absence of the magnetotelluric source problem is due to the fact that dominating periods during significant GIC events are of the order of some minutes. As shown 

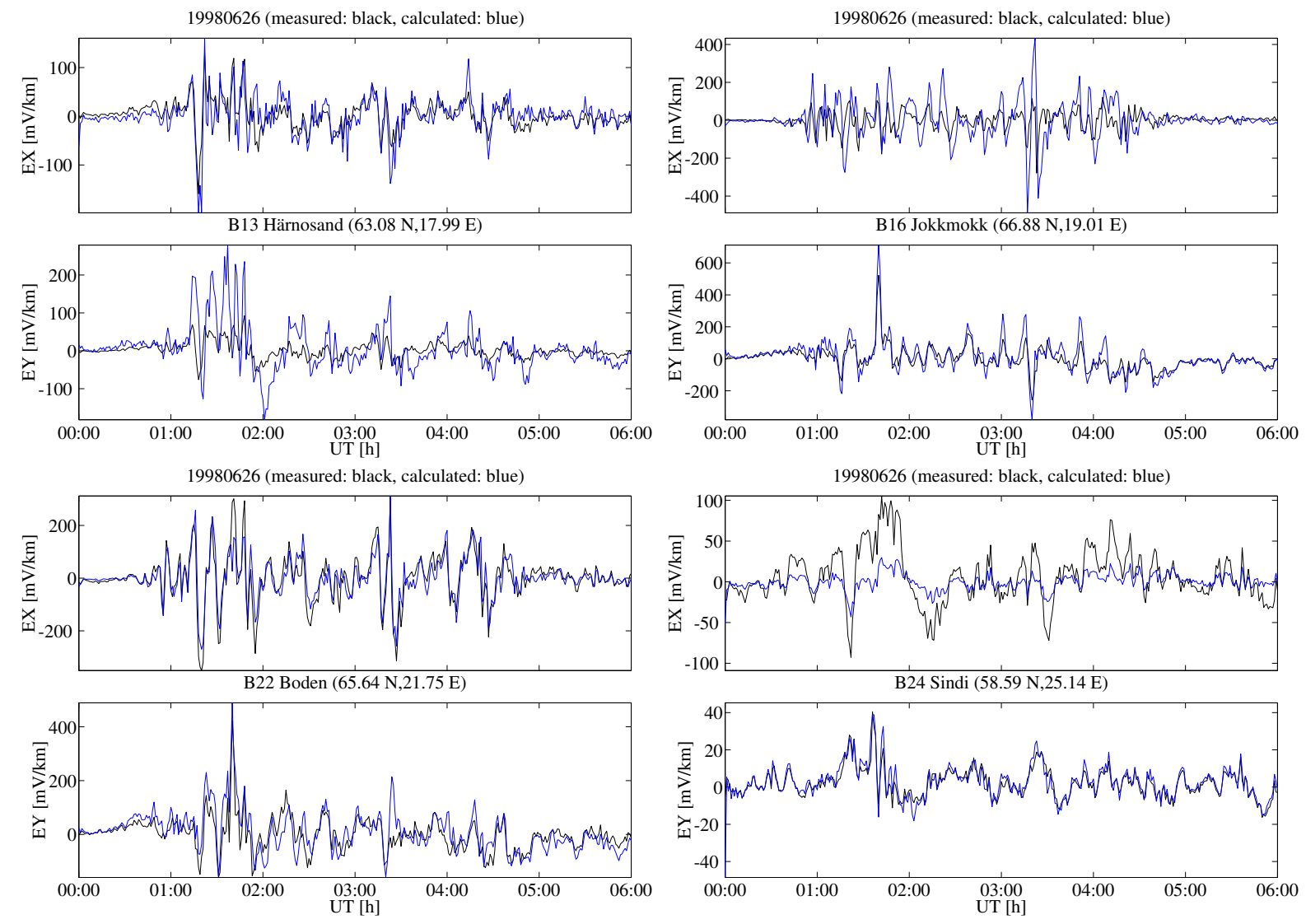

Fig. 5. Measured (black line) and modelled (blue line) electric field at four BEAR sites on 26 June 1998. The local 1-D method (Eq. 3) with conductivity models of Table 1 were used. Each $\left(E_{x}, E_{y}\right)$ pair belongs to the same site named between the panels of $E_{x}$ and $E_{y}$. Locations of the four sites are shown in Fig. 1.

Table 1. Earth conductivity models of selected BEAR sites. Resistivities are given in $\Omega m$. Values below $60 \mathrm{~km}$ are identical at all sites and are shown only in the first column.

\begin{tabular}{rrrrr}
\hline depth $[\mathrm{km}]$ & B13 & B16 & B22 & B24 \\
\hline $0-10$ & 1307 & 949 & 639 & 183 \\
$10-20$ & 755 & 1284 & 377 & 1059 \\
$20-30$ & 583 & 170 & 242 & 1402 \\
$30-40$ & 839 & 86 & 35 & 1509 \\
$40-50$ & 1039 & 102 & 543 & 1668 \\
$50-60$ & 1521 & 105 & 549 & 1322 \\
$60-100$ & 1000 & & & \\
$100-200$ & 300 & & & \\
$200-300$ & 20 & & & \\
$300-400$ & 100 & & & \\
$400-600$ & 20 & & & \\
$600-800$ & 2 & & & \\
$800-1200$ & 5 & & & \\
$1200-1500$ & 0.5 & & & \\
$1500-$ & 1 & & & \\
\hline
\end{tabular}

by numerous theoretical studies, the source effect is small at short periods (e.g. Mareschal, 1986; Osipova et al., 1989).

Measured and modelled geoelectric field and GIC during a very intense storm in April 2001 are shown in Figs. 7-8. The measured GIC at Yllikkälä is at times disturbed probably by a DC railway system in Russia (e.g. the spike just after 18:00 UT). The largest GIC occurred after around 21:30 UT following an auroral breakup. The simultaneous GIC in the natural gas pipeline exceeded 20 A. Until about 21:00 UT, there was a strong eastward current above southern Finland, and later a westward current. The electric field pattern with a clear vortex at 21:30 UT resembles the field due to a westward traveling surge modelled by Viljanen et al. (1999a).

The conductivity model of Table 2 is quite reasonable for southern Finland. For Rauma, the fit is good, and using slightly smaller conductivities further improves the result due to an increased electric field. Concerning Yllikkälä, clearly smaller values of the conductivity would seem necessary. However, the lack of good data from Yllikkälä prevents a detailed analysis.

To show that a modified conductivity model improves model results, we considered 10 stormy days in 1999-2001 (Table 3). There is a high correlation between the measured 

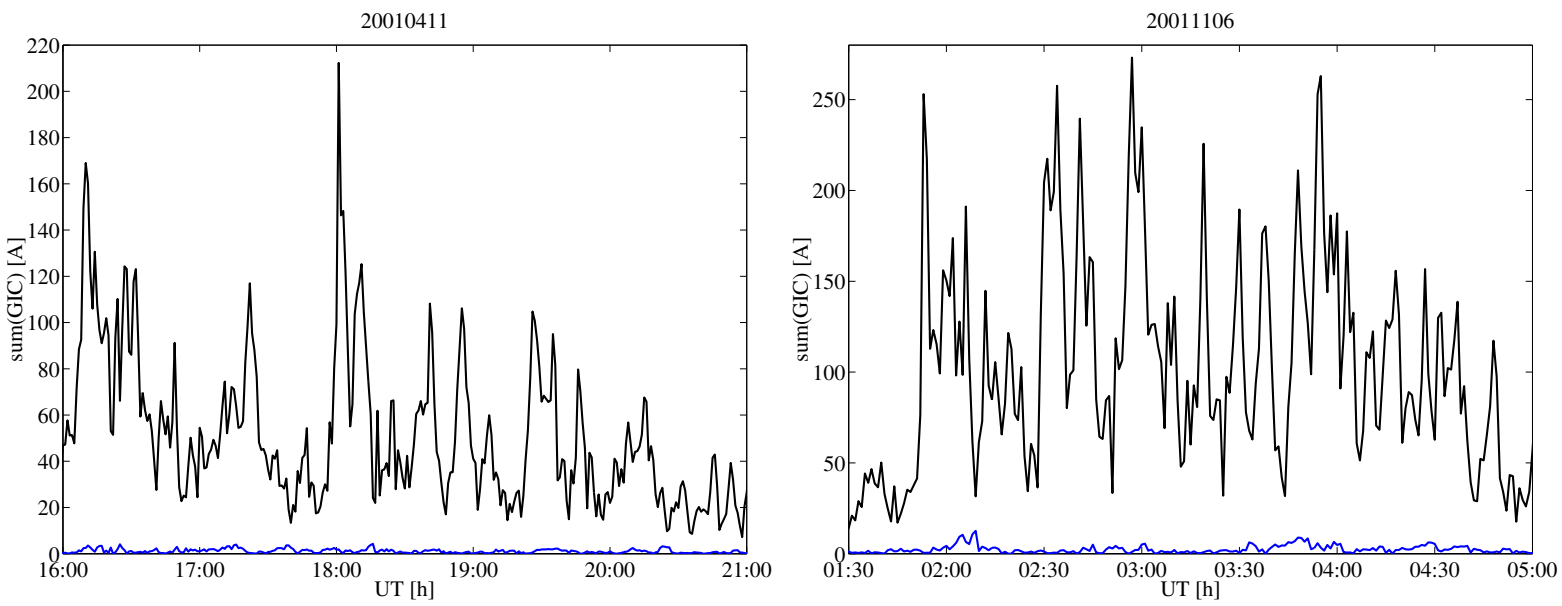

Fig. 6. Comparison of the sum of the absolute values of geomagnetically induced currents in the nodes of the Finnish high-voltage power system (11 April 2001 and 6 November 2001), as calculated with the complex image method (black line) and the local 1-D method. The absolute value of the difference is plotted as a blue line. In both cases, the total horizontal magnetic variation field is the same. The Earth's conductivity model is given in Table 2 .

Table 2. A simple Earth conductivity model used in this study (Viljanen et al., 1999b)

\begin{tabular}{rr}
\hline depth $[\mathrm{km}]$ & resistivity $[\Omega \mathrm{m}]$ \\
\hline $0-3$ & 5000 \\
$3-9$ & 500 \\
$9-14$ & 100 \\
$14-21$ & 10 \\
$21-44$ & 20 \\
$44-150$ & 1000 \\
$150-$ & 1 \\
\hline
\end{tabular}

and modelled values during all events. A linear regression analysis shows that multiplying measured values by 1.8844 yields the best fit when GIC larger than $1 \mathrm{~A}$ is considered. A rough way to modify the Earth model is to divide the conductivities of each layer by $1.8844^{2} \approx 3.55$. This would work exactly with a uniform Earth, since then the electric field is inversely proportional to the square root of the conductivity. With this simple division of all conductivities of the layered model, we obtain the model errors given in Table 4 . The measuring noise is at least $\pm 0.5 \mathrm{~A}$, so the fit is quite reasonable. A more systematic procedure would be necessary for an objective way to modify the Earth model. A division of conductivities by a constant does not necessarily yield the optimal result, but it is obviously necessary to change layer thickness, too.

Taking into account that Rauma is located close to the southern boundary of IMAGE and that the nearest magnetic station Nurmijärvi is at a distance of $200 \mathrm{~km}$, the correspondence between the modelled shape of the GIC curve is good. This is not completely surprising, because the electric field in Fig. 7 varies quite smoothly in a length scale of $100-200 \mathrm{~km}$
Table 3. Stormy events used in the comparison of measured and modelled GIC at Rauma. The second column gives the linear correlation coefficient between the measured and modelled values.

\begin{tabular}{rr}
\hline day & correlation \\
\hline 19990922 & 0.839 \\
20000406 & 0.723 \\
20000715 & 0.922 \\
20000917 & 0.840 \\
20001106 & 0.901 \\
20010331 & 0.925 \\
20010411 & 0.914 \\
20011106 & 0.896 \\
20020907 & 0.860 \\
20021001 & 0.884 \\
\hline
\end{tabular}

in southern Finland. This is in concert with the results by Viljanen et al. (2001), who found that large $d \mathbf{H} / d t$ vectors tend to be north-south oriented in the subauroral region, whereas in the auroral region there is no clear preference of direction. So at subauroral latitudes, there is evidently less spatial variations in ionospheric currents than at higher latitudes.

\subsubsection{Finnish natural gas pipeline}

Calculation of GIC in a pipeline is basically similar to the power system. Two examples in Fig. 9 show that the shape of the modelled curve again follows closely the measured one. This is expected, since the pipeline is located in the same region as the southern part of the power system, and GIC model results are good for it (Fig. 8). Correlation coefficients between the modelled and measured values are mostly larger than 0.8. As discussed by Pulkkinen et al. (2001b), the active cathodic protection system tries to keep the pipe-to-soil volt- 

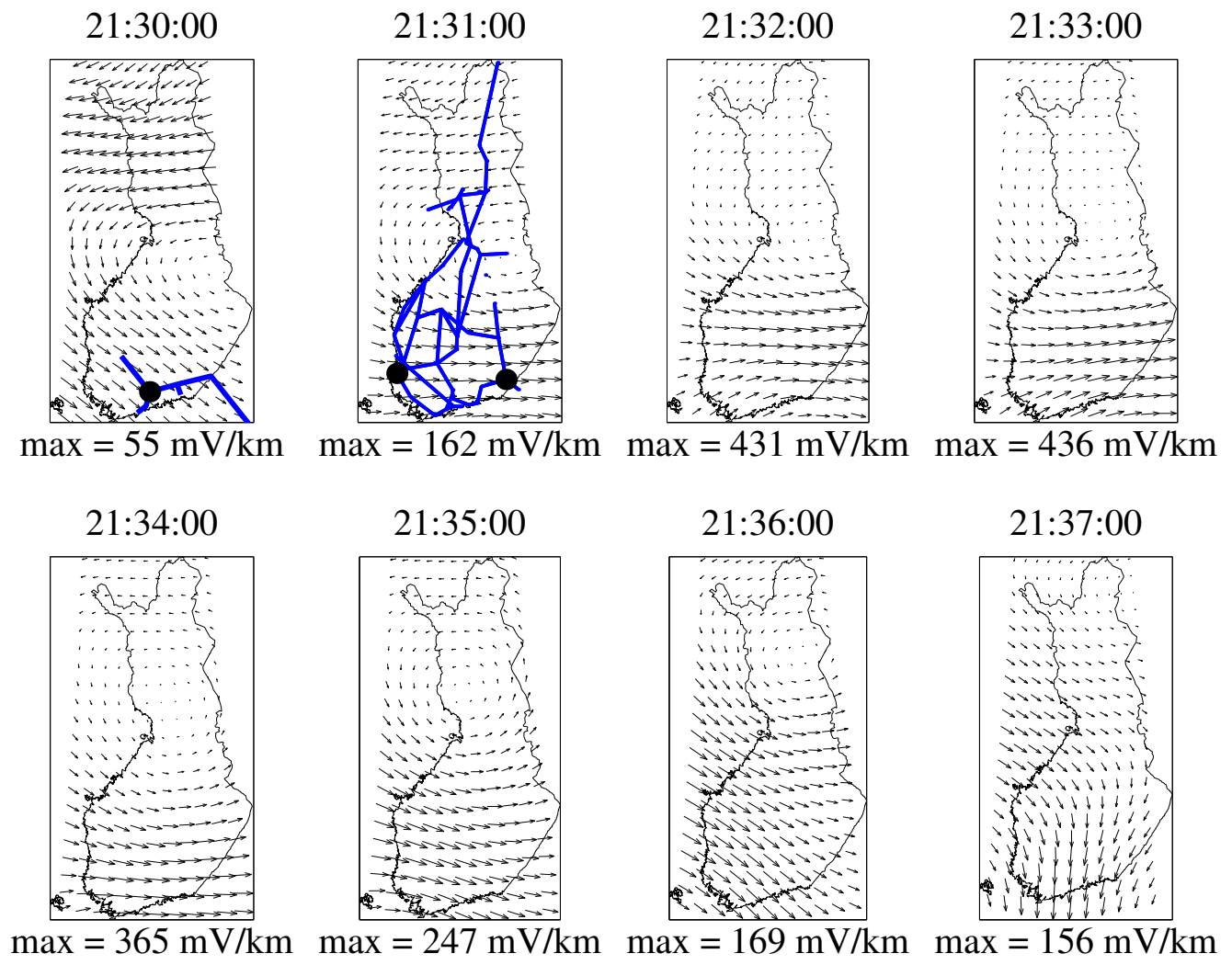

Fig. 7. Snapshots of the calculated electric field on 11 April 2001, using the local 1-D method and the Earth model of Table 2. The main parts of the Finnish natural gas pipeline are sketched in the first plot with blue lines, and the measuring site at Mäntsälä is marked as a black dot. The Finnish $400 \mathrm{kV}$ and $220 \mathrm{kV}$ power lines are shown in the second plot with blue lines. The Rauma and Yllikkälä $400 \mathrm{kV}$ transformers are the black dots in the west and east, respectively.

age constant, so it tends to decrease GIC. The conductivity model happens to be quite suitable, implicitly correcting this bias, too.

\subsubsection{Plane wave model}

In the previous examples, the geoelectric field was calculated using a spatially nonuniform magnetic field. Then the electric field also varies spatially at each time step. It follows that, for example, voltages between nodes in a power system must be integrated separately at each instant. On the other hand, if we can assume that the electric field does not vary spatially then GIC at each time step is

$G I C(t)=a E_{x}(t)+b E_{y}(t)$

where $a$ and $b$ depend on the topology and resistances of the network. This method was used, for example, by Viljanen and Pirjola (1989), who calculated GIC in the Finnish power system using magnetic data of the Nurmijärvi observatory. It turned out that GIC at a site of about $300 \mathrm{~km}$ northeast from the observatory could not be modelled very well. The obvious explanation was that magnetic field variations around the GIC site are usually not sufficiently similar to that at Nurmijärvi.
However, the plane wave method is useful if the electric field is calculated so that the magnetic field is the field observed or interpolated at the GIC site of interest. This means that for each GIC site we select an Earth model used globally. This is slightly different from the local 1-D method discussed above, in which we can use a different model for each Earth surface grid point. The benefit of the plane wave model is that GIC is obtained simply by Eq. (4). Next, we will demonstrate that the plane wave method is really applicable. We use all available GIC data of April, September-November 2001, and also of the other events listed in Table 3.

With the plane wave assumption, GIC at Mäntsälä is $G I C(A)=-70 E_{x}+88 E_{y}$, where the electric field is given in V/km (Pulkkinen et al., 2001b). We now assume that the Earth is uniform. Since we are interested in large currents, we considered only (absolute) values larger than $1 \mathrm{~A}$ when searching for the optimal value of the conductivity. Results are given in Tables 5-6. The conductivity value depends on the way the linear fit is made: the larger value is obtained when we express the measured values as a function of the modelled ones. The smaller value is obtained when we make the fit vice versa. The smaller conductivity yields a better fit for small currents, but for the largest currents the larger conductivity is a better choice. A slightly better fit for large cur- 
Table 4. Misfit of modelled GIC values at Rauma during the events used in Table 3. The first column gives the lower limit of (absolute) GIC values considered. The second column gives the median error of modelled values. The last column gives the number of measured one-minute values larger than $G I C_{0}$. The Earth model is explained in the text

\begin{tabular}{rrr}
\hline $\mathrm{GIC}_{0}$ & median error & $\#$ \\
\hline 1 & $26 \%$ & 2092 \\
2 & $24 \%$ & 709 \\
3 & $23 \%$ & 288 \\
4 & $22 \%$ & 147 \\
5 & $22 \%$ & 93 \\
6 & $23 \%$ & 64 \\
7 & $21 \%$ & 44 \\
8 & $24 \%$ & 33 \\
9 & $27 \%$ & 19 \\
10 & $26 \%$ & 12 \\
\hline
\end{tabular}

rents was found with a conductivity $0.050 \mathrm{ohmm}^{-1}$. So this value could be a reasonable choice for Mäntsälä. Pulkkinen et al. (2001b) used the value $0.031 \mathrm{ohmm}^{-1}$ based on a sample event. Using a properly constructed multi-layer model would evidently provide the best fit, but finding such a model is non-trivial.

GIC at Rauma in the plane wave model is $\operatorname{GIC}(A)=$ $-1.9 E_{x}-22.3 E_{y}$, where the electric field is expressed in $\mathrm{V} / \mathrm{km}$. Now GIC depends practically only on $E_{y}$, which in turn is determined by $d X / d t$. We used the interpolated magnetic field at Rauma (Table 7) or directly the field measured at Nurmijärvi (Table 8). The use of the Nurmijärvi field provides nearly equally good results. There are two reasons for this: Rauma and Nurmijärvi are at about the same latitude, and latitudinal $d X / d t$ variations in the subauroral region are not very strong (Viljanen et al., 2001), cf. also Fig. 7. Nurmijärvi is also the closest magnetometer station to Rauma, so it has the largest effect on the interpolated field. We tested this hypotheses by calculating GIC using the measured magnetic field at Hankasalmi $(62.30 \mathrm{~N}, 26.65 \mathrm{E})$ about $250 \mathrm{~km}$ northeast from Rauma (Table 9). Now the errors are clearly larger, indicating that the time derivative of the magnetic field varies more in the north-south direction than in the east-west direction, at least in the subauroral region.

\subsubsection{Computational performance}

We present here some illustrative numbers of computation times of the full determination of GIC in the Finnish power system. We used a $867 \mathrm{MHz}$ laptop and MatLab by vectorising the code as much as possible, but without using external compiled subroutines. In the following, we consider a oneday event of 1440 one-minute values.

1. Calculation of the ionospheric equivalent currents using the measured ground magnetic field. The number of
Table 5. Misfit of modelled GIC values at Mäntsälä during April, September-November 2001, and during the other events listed in Table 3. The first column gives the lower limit of (absolute) GIC values considered. The second column gives the median error of modelled values. The last column shows the number of measured 10-s values larger than $G I C_{0}$. The electric field was calculated using only the magnetic data of the Nurmijärvi observatory. A uniform Earth model was assumed with a conductivity of $0.07663 \mathrm{ohmm}^{-1}$.

\begin{tabular}{rrr}
\hline $\mathrm{GIC}_{0}$ & median error & $\#$ \\
\hline 2 & $38 \%$ & 17106 \\
4 & $34 \%$ & 5580 \\
6 & $33 \%$ & 2357 \\
8 & $29 \%$ & 1157 \\
10 & $27 \%$ & 650 \\
12 & $26 \%$ & 357 \\
14 & $22 \%$ & 226 \\
16 & $23 \%$ & 152 \\
18 & $22 \%$ & 93 \\
20 & $20 \%$ & 61 \\
22 & $20 \%$ & 35 \\
24 & $24 \%$ & 22 \\
\hline
\end{tabular}

ionospheric grid points was about 400 . Calculation of currents takes about $3 \mathrm{~s}$.

2. Interpolation of the magnetic field at the Earth's surface (three components). Using 231 points to cover Finland takes about $13 \mathrm{~s}$ per day. However, a smaller number of points (around 60) is needed if the plane wave method is used for each power system node separately.

3. Calculation of the electric field at the Earth grid points. With FFT this takes $25 \mathrm{~s}$ for 231 points. (We do not force the time series to be of length $2^{n}$ for an optimal FFT.) Again, the plane wave method would reduce the time due to a smaller number of grid points.

4. Calculation of GIC in the power system nodes and transmission lines. If the electric field varies spatially, then it must be integrated separately for each time step, which takes $137 \mathrm{~s}$ for 62 nodes and 68 lines. The slowness of this phase is mainly due to the need to interpolate the electric field for a numerical integration along transmission lines. However, if the plane wave method is used then GIC is obtained from Eq. (4), which takes a small amount of time.

Altogether, with the local 1-D method, it takes about $3 \mathrm{~min}$ to execute the computation for the Finnish power system for a one-day event using one-minute values. With the plane wave method, it takes less than half a minute. In any case, the computation time is fast enough for an extensive event post analysis.

An operational application is the real-time calculation of GIC in the Finnish natural gas pipeline system within the European Space Agency Space Weather Pilot Programme 

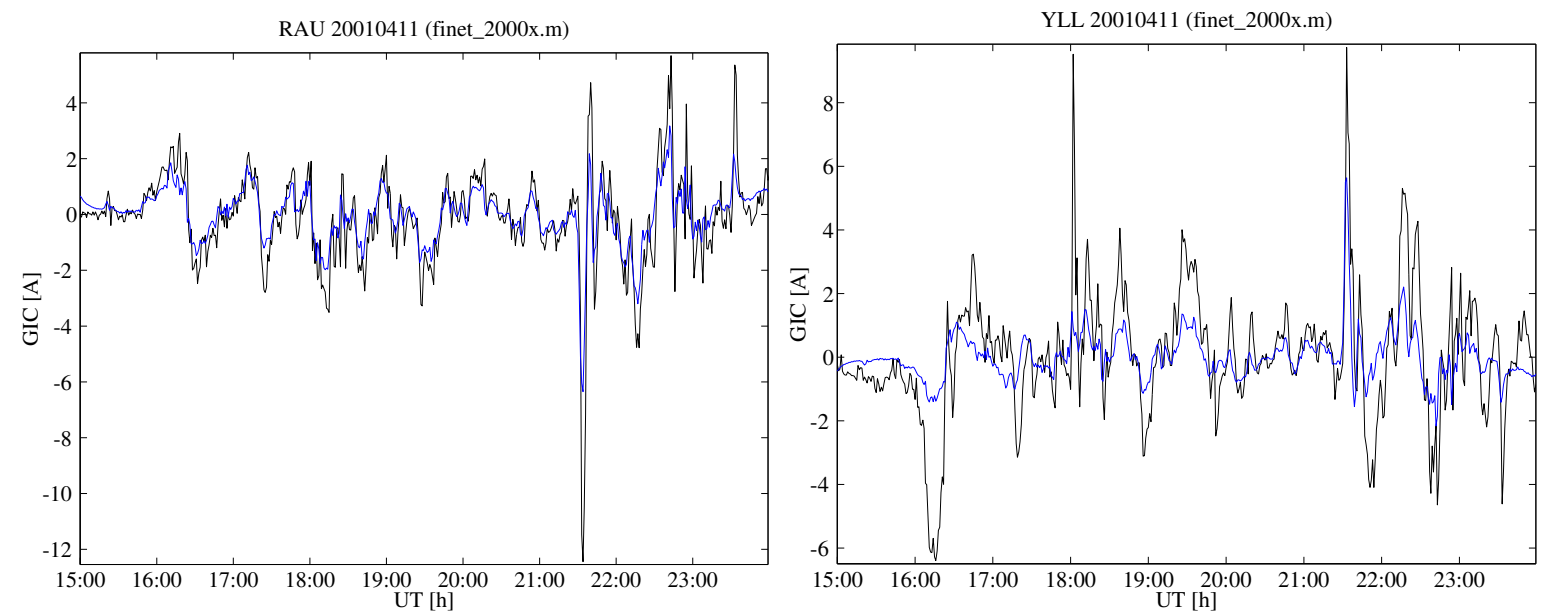

Fig. 8. Measured (black line) and modelled (blue line) geomagnetically induced currents at the Rauma (RAU) and Yllikkälä (YLL) 400 kV transformer stations on 11 April 2001.
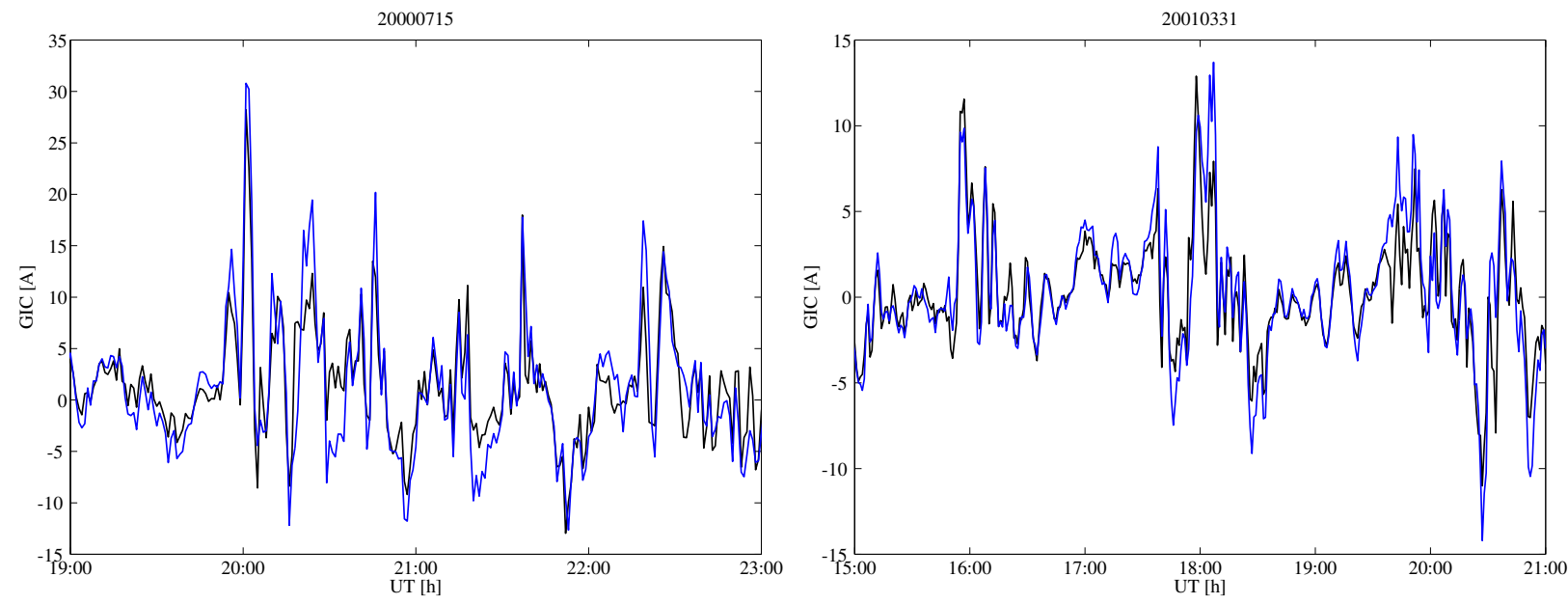

Fig. 9. Measured (black line) and modelled (blue line) geomagnetically induced currents along the Finnish natural gas pipeline at Mäntsälä on 15 July 2000 and on 31 March 2001. The geoelectric field is calculated by the complex image method.

started in spring 2003. The magnetic field data is retrieved from Nurmijärvi and the electric field is calculated assuming that the field does not vary over the pipeline system. As shown in this paper, a fairly good prediction is then expected for GIC. The pipeline company can use the nowcasted GIC as additional information to distinguish between geomagnetic and other reasons for disturbances in the corrosion protection system. Because the magnetic field is used only from one site, the electric field is obtained practically immediately. The same is true for GIC determined with Eq. (4).

\section{Conclusions}

A powerful method to calculate the geoelectric field for space weather purposes is presented in this paper. It needs only two inputs: the horizontal geomagnetic variation field at the
Earth's surface, and 1-D models of the Earth's conductivity. The method of spherical elementary current systems (SECS) allows for interpolating the magnetic field at any ground point. Assuming a planar geometry, the geoelectric field is obtained by the surface impedance relation from the local magnetic field using a local 1-D model of the Earth's conductivity. The validity of the local 1-D method was proved by comparisons of measured and modelled electric fields and geomagnetically induced currents (GIC). Especially concerning GIC, spatially averaged electric fields are obtained with a good accuracy.

A handy way is to consider each GIC site separately, assuming that the magnetic field does not vary spatially, which is the plane wave model. Then GIC is obtained by a multiplication of the horizontal electric field components by constants, which are determined by resistances and the geometry 
Table 6. As Table 5, but with a conductivity of $0.03993 \mathrm{ohmm}^{-1}$

\begin{tabular}{rrr}
\hline $\mathrm{GIC}_{0}$ & median error & $\#$ \\
\hline 2 & $36 \%$ & 17106 \\
4 & $33 \%$ & 5580 \\
6 & $32 \%$ & 2357 \\
8 & $32 \%$ & 1157 \\
10 & $31 \%$ & 650 \\
12 & $30 \%$ & 357 \\
14 & $29 \%$ & 226 \\
16 & $28 \%$ & 152 \\
18 & $27 \%$ & 93 \\
20 & $27 \%$ & 61 \\
22 & $25 \%$ & 35 \\
24 & $20 \%$ & 22 \\
\hline
\end{tabular}

Table 7. Misfit of modelled GIC values at Rauma during the events listed in Table 3. The first column gives the lower limit of (absolute) GIC values considered. The second column gives the median error of modelled values. The last column shows the number of measured one-minute values larger than $G I C_{0}$. Interpolated values of the magnetic field at Rauma were used to calculate the electric field. The conductivity of the uniform Earth was $0.01309 \mathrm{ohmm}^{-1}$.

\begin{tabular}{rrr}
\hline $\mathrm{GIC}_{0}$ & median error & $\#$ \\
\hline 1 & $76 \%$ & 4432 \\
2 & $65 \%$ & 1444 \\
3 & $43 \%$ & 362 \\
4 & $28 \%$ & 169 \\
5 & $23 \%$ & 90 \\
6 & $23 \%$ & 59 \\
7 & $22 \%$ & 42 \\
8 & $22 \%$ & 26 \\
9 & $23 \%$ & 15 \\
10 & $23 \%$ & 10 \\
\hline
\end{tabular}

of the technological conductor system. So this is a somewhat simpler way than that used by Pulkkinen et al. (2000) and Erinmez et al. (2002), in which the electric field must be integrated along conductors separately for each time step. Although the plane wave model neglects the spatial variation of the magnetic field, it provides a good prediction for the electric field in a sufficiently large region around the site under study. In other words, the voltages induced in conductors near the specific site have the largest contribution to GIC at that site. The key point is that the magnetic field must be accurately determined at the GIC site. Some earlier attempts like Viljanen and Pirjola (1989) partly failed due to using the magnetic field measured at a distant location. Now the interpolation of the field by the SECS method fixes this problem.

Dense magnetometer arrays are typically located in sparsely-populated areas in the auroral region. The Finnish natural gas pipeline and most of the high-voltage power system lie in the subauroral area, where there are only some
Table 8. As Table 7, but the magnetic field at Nurmijärvi was used to calculate the electric field.

\begin{tabular}{rrr}
\hline $\mathrm{GIC}_{0}$ & median error & $\#$ \\
\hline 1 & $77 \%$ & 4432 \\
2 & $64 \%$ & 1444 \\
3 & $43 \%$ & 362 \\
4 & $32 \%$ & 169 \\
5 & $26 \%$ & 90 \\
6 & $28 \%$ & 59 \\
7 & $24 \%$ & 42 \\
8 & $26 \%$ & 26 \\
9 & $28 \%$ & 15 \\
10 & $25 \%$ & 10 \\
\hline
\end{tabular}

Table 9. As Table 8, but the magnetic field at Hankasalmi was used to calculate the electric field.

\begin{tabular}{rrr}
\hline $\mathrm{GIC}_{0}$ & median error & $\#$ \\
\hline 1 & $80 \%$ & 4432 \\
2 & $69 \%$ & 1444 \\
3 & $43 \%$ & 362 \\
4 & $35 \%$ & 169 \\
5 & $30 \%$ & 90 \\
6 & $27 \%$ & 59 \\
7 & $28 \%$ & 42 \\
8 & $30 \%$ & 26 \\
9 & $36 \%$ & 15 \\
10 & $36 \%$ & 10 \\
\hline
\end{tabular}

magnetic observation sites. It follows that the spatial accuracy of ionospheric equivalent currents is inevitably smaller than in the auroral region. This is not necessarily a limitation to the usefulness of the calculation method of the electric field, provided that relevant spatial scales are of the same order as magnetometer separations. There is some indication that large subauroral GIC events are more often related to large-scale electrojets than at high latitudes (Viljanen et al., 2001), but further studies are necessary (e.g. Pulkkinen et al., 2003c). From the practical viewpoint, most of the systems vulnerable to GIC are located farther south than the Finnish power grid. So the local 1-D method validated in the subauroral region evidently works well also in mid-latitudes with spatially smoother magnetic fields.

In the future, it may be possible to include detailed 3-D models of the Earth, and perform calculations of the electric field with the multisheet modelling technique, as, for example, by Engels et al. (2002). However, much more computational power is required before such an approach will be feasible for studying large sets of events.

The next step would be using the local 1-D and plane wave methods in GIC forecasting (cf. Kappenman et al., 2000; Erinmez et al., 2002). The ultimate goal in space weather 
research is to provide forecasts in the same way as normal weather forecasts are given today. Concerning the geoelectric field, the ground magnetic variation field should be predicted. Today's skills are not yet good enough for really accurate forecasting, but there are rapidly developing efforts to improve this in the way required in GIC forecasting (Gleisner and Lundstedt, 2001; Valdivia et al., 1999; Weigel et al., 2002).

The method described in this paper is also applicable to magnetotelluric studies. Its first step includes an equivalent description of ionospheric currents, so it gives full control on the source field. Second, Earth models can be directly tested with the local 1-D method for any events. GIC are related to a spatially smoothed electric field, so they yield information on Earth's conductivity in a regional scale, and the 1-D assumption seems then reasonable, as in the southern Finland area considered in this paper.

Acknowledgement. The Academy of Finland supported the works of O.A. and A.P. We thank all institutes maintaining the IMAGE magnetometer network. INTAS funded partly the BEAR project (no. 97-1162). Fingrid Oyj and Gasum Oy are acknowledged for a fruitful co-operation on geomagnetically induced currents in the Finnish high-voltage power system and in the Finnish natural gas pipeline, respectively.

The Editor in Chief thanks I. Ferguson and another referee for their help in evaluating this paper.

\section{References}

Amm, O.: Ionospheric Elementary Current Systems in Spherical Coordinates and Their Application, J. Geomag. Geoelectr., 49, 947-955, 1997.

Amm, O. and Viljanen, A.: Ionospheric disturbance magnetic field continuation from the ground to the ionosphere using spherical elementary current systems, Earth Planets Space, 51, 431-440, 1999.

Bolduc, L.: GIC observations and studies in the Hydro-Quebec power system, J. Atmos. Sol.-Terr. Phys., 64, 1793-1802, 2002.

Boteler, D. H. and Pirjola, R. J.: The complex-image method for calculating the magnetic and electric fields produced at the surface of the Earth by the auroral electrojet, Geophys. J. Int., 132, 31-40, 1998.

Boteler, D. H., Pirjola, R. J., and Nevanlinna, H.: The effects of geomagnetic disturbances on electrical systems at the Earth's surface, Adv. Space Res., 22, 17-27, 1998.

Dmitriev, V. and Berdichevsky, M.: The fundamental model of magnetotelluric sounding, IEEE Proc., 67, 1034, 1979.

Engels, M., Korja, T., and the BEAR Working Group: Multisheet modelling of the electrical conductivity structure in the Fennoscandian Shield, Earth Planets Space, 54, 559-573, 2002.

Erinmez, I. A., Kappenman, J. G., and Radasky, W. A.: Management of the geomagnetically induced current risks on the national grid company's electric power transmission system, J. Atmos. Sol.-Terr. Phys., 64, 743-756, 2002.

Gleisner, H. and Lundstedt, H.: A neural network-based local model for prediction of geomagnetic disturbances, J. Geophys. Res., 106, 8425-8433, 2001.

Gummow, R. A.: GIC effects on pipeline corrosion control systems, J. Atmos. Sol.-Terr. Phys., 64, 1755-1764, 2002.
Kappenman, J. G.: Geomagnetic Storms and Their Impact on Power Systems, IEEE Power Engineering Review, May 1996, 5-8, 1996.

Kappenman, J. G., Radasky, W. A., Gilbert, J. L., and Erinmez, I. A.: Advanced Geomagnetic Storm Forecasting: A Risk Management Tool for Electric Power System Operations, IEEE T. Plasma Sci., 28, 2114-2121, 2000.

Korja, T., Engels, M., Zhamaletdinov, A. A., Kovtun, A. A., Palshin, N. A., Smirnov, M. Yu., Tokarev, A. D., Asming, V. E., Vanyan, L. L., Vardaniants, I. L., and the BEAR Working Group: Crustal conductivity in Fennoscandia - a compilation of a database on crustal conductance in the Fennoscandian Shield, Earth Planets Space, 54, 535-558, 2002.

Lahtinen, M. and Elovaara, J.: GIC Occurrences and GIC Test for 400 kV System Transformer, IEEE T. Power Delivery, 17, 555561, 2002.

Lehtinen, M. and Pirjola, R.: Currents produced in earthed conductor networks by geomagnetically-induced electric fields, Ann. Geophysicae, 3, 479-484, 1985.

Lesher, R. L., Porter, J. W., and Byerly, R. T.: SUNBURST - A Network of GIC Monitoring Systems, IEEE T. Power Deliver., 9, 128-137, 1994.

Lindell, I. V., Hänninen, J. J., and Pirjola, R.: Wait's ComplexImage Principle Generalized to Arbitrary Sources, IEEE T. Antennas Propagat., 48, 1618-1624, 2000.

Mareschal, M.: Modelling of natural sources of magnetospheric origin in the interpretation of regional induction studies: a review, Surv. Geophys., 8, 261-300, 1986.

Molinski, T. S.: Why utilities respect geomagnetically induced currents, J. Atmos. Sol.-Terr. Phys., 64, 1765-1778, 2002.

Osella, A., Favetto, A., and Lopez, E.: Currents induced by geomagnetic storms on buried pipelines as a cause of corrosion, J. Appl. Geophys., 38, 219-233, 1998.

Osipova, I. L., Hjelt, S. E., and Vanyan, L. L.: Source field problems in northern parts of the Baltic Shield, Phys. Earth Planet. Int., 53, 337-342, 1989.

Pirjola, R. and Viljanen, A.: Complex image method for calculating electric and magnetic fields produced by an auroral electrojet of a finite length, Ann. Geophysicae, 16, 1434-1444, 1998.

Pirjola, R., Pulkkinen, A., and Viljanen, A.: Studies of Space Weather Effects on the Finnish Natural Gas Pipeline and on the Finnish High-Voltage Power System, accepted for publication in Adv. Space Res., 2002.

Pulkkinen, A., Viljanen, A., Pirjola, R., and BEAR Working Group: Large geomagnetically induced currents in the Finnish highvoltage power system, Finn. Meteorol. Inst. Rep., 2000:2, 99 pp., 2000.

Pulkkinen, A., Pirjola, R., Boteler, D., Viljanen, A., and Yegorov, I.: Modelling of space weather effects on pipelines, J. Appl. Geophys., 48, 233-256, 2001a.

Pulkkinen, A., Viljanen, A., Pajunpää, K., and Pirjola, R.: Recordings and occurrence of geomagnetically induced currents in the Finnish natural gas pipeline network, J. Appl. Geophys., 48, 219-231, 2001b.

Pulkkinen, A., Amm, O., Viljanen, A., and BEAR Working Group: Ionospheric equivalent current distributions determined with the method of spherical elementary current systems, J. Geophys. Res., 108(A2), 1053, doi:10.1029/2001JA005085, 2003 a.

Pulkkinen, A., Amm, O., Viljanen, A., and BEAR Working Group: Separation of the geomagnetic variation field into external and internal parts using the spherical elementary current system method, Earth Planets Space, 55, 117-129, $2003 \mathrm{~b}$. 
Pulkkinen, A., Thomson, A., Clarke, E., and McKay, A.: April 2000 storm: ionospheric drivers of large geomagnetically induced currents, Ann. Geophysicae, 21, 709-717, 2003c.

Tanskanen, E. I., Viljanen, A., Pulkkinen, T. I., Pirjola, R., Häkkinen, L., Pulkkinen, A., and Amm, O.: At substorm onset, $40 \%$ of AL comes from underground, J. Geophys. Res., 106, 13 119-13 134, 2001.

Thomson, D. J., and Weaver, J. T.: The Complex Image Approximation for Induction in a Multilayered Earth, J. Geophys. Res., 80, 123-129, 1975.

Trichtchenko, L. and Boteler, D. H.: Modelling of geomagnetic induction in pipelines, Ann. Geophysicae, 20, 1063-1072, 2002.

Valdivia, J. A., Vassiliadis, D., Klimas, A., and Sharma, A. S.: Modeling the spatial structure of the high latitude magnetic perturbations and the related current systems, Physics of Plasmas, 6, 4185-4194, 1999.

Viljanen, A. and Pirjola, R.: Statistics on geomagnetically-induced currents in the Finnish $400 \mathrm{kV}$ power system based on recordings of geomagnetic variations. J.Geomagnetism and Geoelectricity,
41, 411-420, 1989.

Viljanen, A., Amm, O., and Pirjola, R.: Modelling Geomagnetically Induced Currents During Different Ionospheric Situations, J. Geophys. Res., 104, 28 059-28 072, 1999a.

Viljanen, A., Pirjola, R., and Amm, O.: Magnetotelluric source effect due to 3-D ionospheric current systems using the complex image method for 1-D conductivity structures, Earth Planets Space, 51, 933-945, 1999b.

Viljanen, A., Nevanlinna, H., Pajunpää, K., and Pulkkinen, A. Time derivative of the horizontal geomagnetic field as an activity indicator, Ann. Geophysicae, 19, 1107-1118, 2001.

Wait, J. R. and Spies, K. P.: On the image representation of the quasi-static fields of a line current source above the ground, Can J. Phys., 47, 2731-2733, 1969.

Weigel, R. S., Vassiliadis, D., and Klimas, A. J.: Coupling of the solar wind to temporal fluctuations in ground magnetic fields, Geophys. Res. Lett., 29, No. 19, doi:10.1029/2002GL014740, 2002. 